\title{
Therapeutic Outcomes Of Functional Strength Training Versus Conventional Physical Therapy In Children With Cerebral Palsy: A Comparative Study
}

Amira M Abd-Elmonem* and Walaa A Abd El-nabie

*Correspondence: Dramira.salim2020@gmail.com

CrossMark

$\leftarrow$ Click for updates

Lecturer, Physical Therapy for Paediatrics Department, Faculty of Physical Therapy, Cairo University, Egypt.

\begin{abstract}
Background: Conventional physical therapy has been used in children with cerebral palsy aiming for improving motor abilities and functional independence. Resistance training is now commonly used in clinical practice in children and adolescents with spastic cerebral palsy.

Purpose: To investigate and compare the effects of functional strength training versus conventional physical therapy in children with cerebral palsy.

Methods: Fifty-three children with spastic diplegia age ranged from 7 to 11 years were included. They were randomly assigned to either group I (conventional physical therapy) or group II (functional strength training). The training was conducted for 90 minutes three-times a week for 6 successive months. Selective voluntary motor control, gross motor function and functional balance were assessed before and immediately after the intervention using selective control assessment of lower extremity, gross motor function measure and pediatric balance scale.
\end{abstract}

Results: The pre-treatment comparison showed non-significant difference between the two groups in all measured variables. Within group comparison showed significant improvement in the mean values of selective motor control, gross motor function and functional balance in the two groups. While posttreatment comparison revealed significant greater improvements in the group II compared with the group I $(\mathrm{P}<0.05)$.

Conclusion: These outcomes indicated that functional strength training is likely more effective than conventional physical therapy in improving selective voluntary motor control, gross motor function and functional balance.

Keywords: Cerebral palsy, Functional balance, Functional strengthening exercises, Selective voluntary motor control, Spastic diplegia

\section{Introduction}

Cerebral palsy (CP) is a complex and heterogeneous condition resulting from lesions in the immature brain referred as unprogressive chronic encephalopathy characterized primarily by disorder of movement and posture [1-3]. Although the brain damage is irreversible and unprogressive, the clinical manifestations progress, lead to structural and morphological changes of the musculoskeletal system such as muscle contractures and bone deformation. Spastic diplegia is a highly prevalent type of CP that prohibits the motor control of lower limbs and may also affect the axial parts and upper extremities depending on the severity of the condition [4]. Brain scans findings commonly reveal injury to periventricular white matter (PWM). Damage to the corticospinal tracts (CSTs) within the PWM has been associated with motor disability and impaired selective voluntary motor control problems (SVMC) [5]. The term SVMC has been 
defined as the capacity to isolate the activation of muscles in a desired pattern by voluntary movement or posture. Although spasticity and contractures are considered the main causes for impairments, deficits in SVMC are the main contributor to motor impairments [6-8].

Delayed or abnormal development of the postural-balance mechanisms and delayed gross motor development are common features in all types of $\mathrm{CP}$ with inability to develop more mature neurological postural mechanisms [9]. Balance impairment in spastic diplegia may be due to difficulty in activating and timing muscle contraction and muscle weakness secondary to inactivity [10].

Progressive resistance training has been shown to improve muscle strength and the effort associated with resistance training does not exacerbate spasticity in subjects with $\mathrm{CP}$ [11]. It has been recognized to provoke strength gains through progressive increase of the loads depending on the subject's abilities and determined by the repetition maximum (RM). One $\mathrm{RM}$ is the maximum load the subject can perform an exercise for one complete repetition correctly [12].

Previous studies investigated the effects of resistance training in children with $\mathrm{CP}$ and reported positive influences on gait, gross motor function, muscle strength, spasticity and equilibrium [1,13-15]. However, no studies to date have focused on the effects of either progressive functional strength training training or conventional physiotherapy on SVMC. Here, we compared the effects of functional strength training versus conventional physical therapy on SVMC, gross motor function and functional balance in children with spastic diplegia.

\section{Materials and methods Study design}

A randomized-controlled trial was conducted between 2015 and 2018 in accordance with the Code of Ethics of the World Medical Association (Declaration of Helsinki). Participants' parents/ legal guardians provided written informed consent before the study was conducted.

\section{Participants}

A convenient sample of children with $\mathrm{CP}$ was recruited from the Outpatient Clinic, Faculty of Physical Therapy Cairo University. They have been screened by the principal investigator and included if they were 1) diagnosed as CP spastic diplegia, 2) aged from 7 to 11 years, 3) grade 1 and $1+$ spasticity according to the Modified Ashworth scale [16], 4) at level I and II of motor function according to the gross motor function classification system-extended and revised (GMFCS-ER) [17], 5) able to understand, follow instructions and participate in a group training program. Participants have been excluded if they, 1) had significant mental or behavioral disorders, 2) significant visual or auditory problems according to medical reports (audio-vestibular and ophthalmic examination), 3) structural or fixed soft tissue deformities of the lower extremities, 4) undergone musculoskeletal surgery or botulinum toxin injection in the lower limbs in past six months.

\section{Sample size estimation}

Sample size calculation was performed prior to the study using G*POWER statistical software (version 3.1.9.2; Franz Faul, Universitat Kiel, Germany) [F tests- MANOVA: Repeated measures, within-between interaction, $\alpha=0.05, \beta=0.2$, Pillai $V=0.13$, and large effect size] and revealed that the appropriate sample size for this study was $\mathrm{N}=25$ children in each group. We recruited up to 60 children to account for the possible withdrawal rates.

\section{Randomization}

Following the baseline measurements, a randomization process was performed for 60 children using sealed opaque envelopes. The investigator prepared 60 closed envelopes, with each envelope containing a card labeled as either control or study. Each child/legal guardian was asked to draw a closed envelope that determined whether he/she was allocated to group I ( $\mathrm{N}=27,14$ boys and 13 girls) or group II ( $\mathrm{N}=26,14$ boys and 12 girls). After dropout, only 53 children completed the study. See participants flow chart Figure 1.

Children in the group I received a conventional physical therapy program whereas those in the group II received a functional strength training. The treatment procedures were for conducted 90 minutes three-times a week for 6 successive months for the two groups.

\section{Procedures}

Weight and height were recorded using a calibrated floor scale (ZT-120 model), Hangzhou Tianheng Technology Co. Ltd (Hangzhou, China). Each child was evaluated for SVMC, gross motor function and functional balance before and at the end of the six months of treatment by the same examiner who was blinded in terms of the group to which each child was assigned.

\section{Primary outcome measure \\ Selective voluntary motor control:}

The selective control assessment of lower extremity (SCALE) is a valid and reliable tool frequently used for assessment of SVMC in children with spastic CP. It is currently used to evaluate the isolated movements of the lower limb joints bilaterally and doesn't require specialized equipment. It can be completed within 15 minutes with a maximum score of 20 points, 10 points for each limb. The participants were asked to execute specific movement patterns using a three-second verbal count including, (1) hip flexion and extension with the knee extended, (2) knee extension and flexion, (3) ankle dorsiflexion and plantarflexion with the knee extended, (4) subtalar inversion and eversion, and (5) toe flexion and extension. The assessment procedures were carried out from the sitting position for all joints except for the hip joint which was performed side-lying with the limb supported by the examiner $[\mathbf{7}, 18]$. 


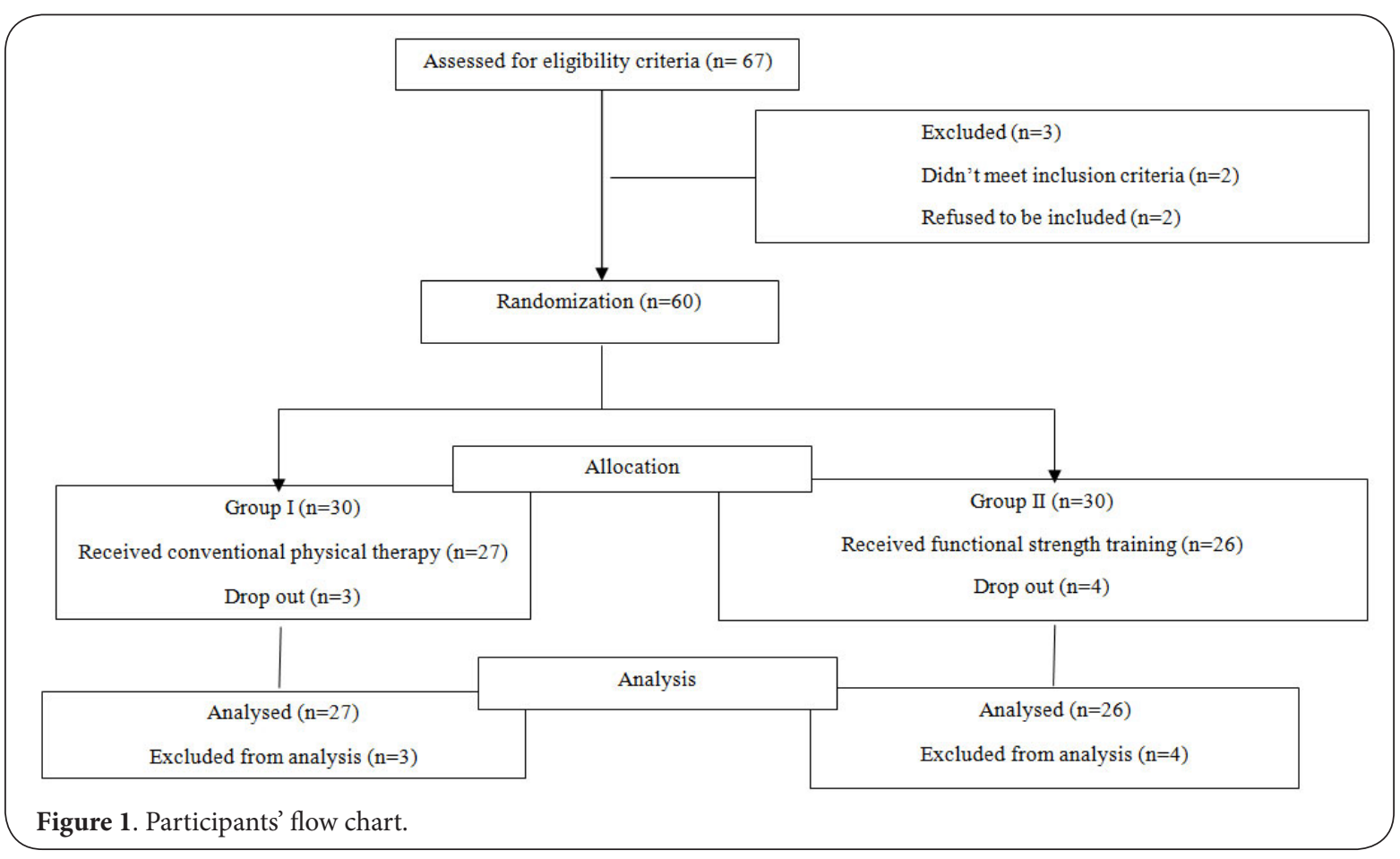

The examiner demonstrated the desired movement by passively moving the limb. Next, the child was instructed to accomplish the task at the same speed without movement of the other joints of the tested limb or the contralateral limb (mirror action). Then, the passive range of motion of each joint was noted and compared with the observed active range and scored as "normal" ( 2 points), "impaired" (1 points), and "unable" (0 points). When the child couldn't initiate the desired movement or performed a synergistic mass flexor or extensor pattern a grade of "unable" was given. A grade of "impaired" was assigned if he/she performed isolated joint movement but with errors including; only one directional movement, less than $50 \%$ of movement accomplished, movement of non-examined joints (including mirror movements), or more than three-second verbal count. The grade of "normal" was given when the desired movement was performed within the three-second verbal count without movement of untested joints $[5,8,18]$.

\section{Secondary outcome measure Gross motor function}

The gross motor function measure-88 (GMFM-88) was used to evaluate the motor function. It is a valid and reliable criterionreferenced instrument that was created to assess the change in gross motor function over time in individuals with $C P$ [17]. It consists of 5 sections including; A) lying and rolling, B) sitting, C) crawling and kneeling, D) standing; E) walking, running, jumping. Dimensions ' $D$ ' and ' $E$ ' were selected as goal areas in the current study. Each item was scored on a four-point scale as $0,1,2,3$ by observing his/her performance with higher scores representing a better performance. The scoring key was; $0=$ does not initiate, $1=$ initiates the task $(<10 \%), 2=$ partial completion of the task ( $10 \%$ to $<100 \%), 3=$ task completed (100\%). If an item was not tested it was marked as not tested [3].

\section{Functional balance}

The pediatric balance scale (PBS) is a valid and reliable modified form of the Berg Balance Scale that is used to evaluate functional balance in children with CP with mild to severe motor impairment [18]. It assesses the child's performance of the functional activities in 3 dimensions, including sitting, standing, and postural change. The assessment takes about 15 minutes without any specialized equipment. The assessment was conducted in accordance with the PBS manual detailed instructions. Each item was graded from 0 to 4 points giving a maximum total score 56 points, with a higher score representing a better performance [7].

\section{Intervention}

The treatment was conducted by qualified, experienced physical therapists. All children in the two groups received 3 sessions per week for six successive months. Each session lasted for 90 minutes. Remaining 3 days of the week, all children were instructed to perform a home routine program leaving a day off for rest.

Group I: The 27 children (14 boys and 13 girls) in this group received conventional physical therapy program based on neurodevelopmental approach. The physical therapy program focused on reinforcement of normal motor development, facilitation of postural stability and balance, stimulation 
of righting and equilibrium reactions to improve postural mechanism, closed and open environment gait training, and maintenance of soft tissue and joint mobility training [19].

Group II: The 26 children ( 14 boys and 12 girls) in this group received a functional strength training which replaced their conventional physical therapy program. The training sessions were held in circuit training of small groups including 4 to 6 children supervised by two qualified, experienced physical therapists per group. These exercises included sit-to-stand exercise, lateral/forward step up exercise (i.e. climbing a stair or stepping up onto a kerb), and half-knee rise exercise (i.e. rising from the ground) loaded with a weighted vest $[1,12]$. All exercises were performed in 1-3 sets of 10-15 repetitions, with a 90 second rest in between the sets. Each exercise was performed within 7-10 minutes. The training load for the exercises was established according to the individual eightrepetition maximum ( $8 \mathrm{RM}$ ) test. The $8 \mathrm{RM}$ is approximately equal to $80 \% 1 \mathrm{RM}$. Free weights were used to provide resistance using adjustable weight cuffs with Velcro straps and weight vest. During the training, intensity progressively increased, based on repeated estimation of the 8 RM. Each session included stretching exercises, closed and open environment gait training in addition to functional strength training that consisted of sit-to-stand, lateral/forward step up and half-knee rise exercises.

- Sit-to-stand bilateral exercises were performed from sitting on a chair (no armrests, no backrest) with the hips at $90^{\circ}$ and knees at $105^{\circ}$ flexion (thigh parallel to the floor). One standup / sit-down was considered as one repetition. For those who could not perform the exercise, they were allowed to decrease knee flexion to $100^{\circ}-120^{\circ}$ (hips slightly higher than knees by raising the seat of the chair. He/she was instructed to move the trunk forward by flexion of the hips until the shoulders are above the knee joints and stand up for one second then to sit down.

-Lateral/forward step up unilateral exercises was achieved from standing at about $10 \mathrm{~cm}$ besides or in front of the step (40 - 20-centimeter height) with erect trunk and hips at neutral position. The target limb was flexed in hip and knee, foot placed on step while the other limb was fully extended at hip and knee (as far as possible, full extension is defined as $0^{\circ}$ ). The child was asked to maintain the foot as flat on the floor as possible. The lower extremities were trained alternatively. One step up/ one step down was considered as one repetition. He/she was instructed to place the right foot up on the step, next to the left foot and extend the knees as much as possible and keep standing with both feet on the step for 1 second. Then to place the right foot down on the floor, leave left foot up on the step.

-Half knee rise was performed with the child on the floor (or mat) in half-kneeling position on target knee (i.e left knee). $\mathrm{He} / \mathrm{she}$ was asked to place the hands-on waist or knee with the trunk erect or slightly forward. The left hip was in $20^{\circ}$ flexion to neutral position (as long as buttocks are clear of lower legs) while the knee on floor in $90^{\circ}-130^{\circ}$ flexion and the foot on the floor. The right hip in $70^{\circ}-110^{\circ}$ flexion and the knee was in $70^{\circ}-90^{\circ}$ flexion and the foot as flat on floor as possible, preferably heel contact. One standup/one return was considered as one repetition. The child was asked to move the trunk forward by flexion of the hips until the shoulders are above the right knee joint then stand up while keeping weight on the leading leg standing for one second (If possible, place the left foot next to the right). Then he was instructed to return to half-knee position.

\section{Data analysis}

Descriptive and t-test were conducted for comparison of subject characteristics between both groups. Chi- squared test was used for comparison of sex distribution between groups. Normal distribution of data was checked using the ShapiroWilk test for all variables. Levene's test for homogeneity of variances was conducted to test the homogeneity between groups. Mixed MANOVA was performed to compare within and between groups effects on SCALE, GMFM-88 and PBS. Partial squared eta was considered as the effect size. Posthoc tests using the Bonferroni correction were carried out for subsequent multiple comparison. The level of significance for all statistical tests was set at $p<0.05$. All statistical analysis was conducted through the statistical package for social studies (SPSS) version 22 for windows (IBM SPSS, Chicago, IL, USA).

\section{Results}

\section{Participants' characteristics}

Table 1 showed the subject characteristics of both groups. There was no significant difference between both groups in the mean age, weight, height and BMI $(p<0.05)$. Also, there was no significant difference in sex distribution between groups $(p=0.88)$.

\section{Effect of treatment on SVMC, gross motor function and functional balance}

Mixed MANOVA revealed that there was a significant interaction of treatment and time (Wilks' Lambda $=0.02 ; \mathrm{F}(5,47)=$ 309.74, $\left.\mathrm{p}=0.001, \eta^{2}=0.97\right)$. There was a significant main effect of time (Wilks' Lambda $=0.009 ; F(5,47)=1043.64, p=0.001, \eta^{2}$ $=0.99$ ). There was a significant main effect of treatment (Wilks' Lambda=0.16; $\left.F(5,47)=48.89, p=0.001, \eta^{2}=0.83\right)$. Table 2

Table 1. Comparison of the mean age, weight, height, BMI and sex distribution between group I and II.

\begin{tabular}{llllll}
\hline & Group I & Group II & & & \\
\hline & $\overline{\mathrm{x}} \pm$ SD & $\overline{\mathrm{x}} \pm$ SD & MD & t- value & p-value \\
\hline Age (years) & $8.18 \pm 1.03$ & $7.8 \pm 1.05$ & 0.38 & 1.32 & $0.19^{*}$ \\
Weight $(\mathrm{kg})$ & $27.6 \pm 4.06$ & $27.15 \pm 3.68$ & 0.45 & 0.42 & $0.67^{\star}$ \\
Height $(\mathrm{cm})$ & $120.07 \pm 5.71$ & $120.53 \pm 6.52$ & -0.46 & -0.27 & $0.78^{*}$ \\
BMI $\left(\mathrm{kg} / \mathrm{m}^{2}\right)$ & $19.21 \pm 3.08$ & $18.75 \pm 2.69$ & 0.46 & 0.57 & $0.56^{*}$ \\
Boys/Girls & $14 / 13$ & $14 / 12$ & & $\left(\boldsymbol{\chi}^{2}=0.02\right)$ & $0.88^{*}$ \\
\hline
\end{tabular}

$\overline{\mathrm{x}}$, Mean; SD: Standard deviation; MD: mean difference; $\chi 2$ : Chi squared value; $p$-value: Level of significance;

${ }^{\star}$ : Non-significant 
Abd-Elmonem et al, Physical Therapy and Rehabilitation 2019, http://www.hoajonline.com/journals/pdf/2055-2386-6-7.pdf

Table 2. Pre and post-treatment mean values of the SCALE, GMFM-88 and PBS in group I and II.

\begin{tabular}{|c|c|c|c|c|c|c|c|c|}
\hline & \multicolumn{3}{|l|}{ Pre } & \multicolumn{3}{|l|}{ Post } & \multicolumn{2}{|c|}{ Pre vs post } \\
\hline & Group I & Group II & & Group I & Group II & & Group I & Group II \\
\hline & $\overline{\mathrm{X}} \pm \mathrm{SD}$ & $\overline{\mathrm{X}} \pm \mathrm{SD}$ & P-value & $\overline{\mathrm{X}} \pm \mathrm{SD}$ & $\overline{\mathrm{X}} \pm \mathrm{SD}$ & P-value & P-value & P-value \\
\hline \multicolumn{9}{|l|}{ SCALE } \\
\hline Right lower extremity & $2.44 \pm 0.8$ & $2.23 \pm 0.9$ & $0.39^{\star}$ & $2.81 \pm 0.92$ & $6.5 \pm 1.17$ & $0.001^{\star *}$ & $0.02^{\star \star}$ & $0.001^{\star *}$ \\
\hline Left lower extremity & $2.92 \pm 0.78$ & $2.61 \pm 0.75$ & $0.14^{*}$ & $3.29 \pm 0.77$ & $6.8 \pm 0.74$ & $0.001^{\star *}$ & $0.001^{\star *}$ & $0.001^{\star *}$ \\
\hline \multicolumn{9}{|l|}{ GMFM-88 (\%) } \\
\hline Dimension D & $61.04 \pm 1.95$ & $60.11 \pm 2.61$ & $0.14^{*}$ & $68.73 \pm 2.08$ & $80.45 \pm 2.5$ & $0.001^{\star *}$ & $0.001^{* *}$ & $0.001^{\star *}$ \\
\hline Dimension E & $31 \pm 2$ & $31.84 \pm 2.4$ & $0.17^{*}$ & $37.75 \pm 2.33$ & $47.4 \pm 2.92$ & $0.001^{\star *}$ & $0.001^{\star *}$ & $0.001^{\star \star}$ \\
\hline PBS & $32.3 \pm 4.6$ & $32.42 \pm 3.63$ & $0.91^{*}$ & $37.44 \pm 4.26$ & $46 \pm 2.34$ & $0.001^{* *}$ & $0.001^{\star \star}$ & $0.001^{\star *}$ \\
\hline
\end{tabular}

$\overline{\mathrm{x}}$ : Mean; SD: Standard deviation; p-value: level of significance; ${ }^{\star}$ : Non-significant; ${ }^{* *}$ : Significant

showed descriptive statistics of SCALE, GMFM-88 and PBS and the significant level of comparison between groups as well as significant level of comparison between before and after treatment in each group.

\section{Between group comparison}

There was no significant difference between the groups I and II in all parameters before treatment ( $p>0.05)$. After treatment there was a significant increase in the right and left extremities SCALE, dimension D and E of GMFM-88 and PBS of the group II compared with that of group I ( $p>0.001)$.

\section{Within group comparison}

There was a significant increase in the right and left extremities SCALE, dimension D and E of GMFM-88 and PBS after treatment compared with that before treatment in both group I and II $(\mathrm{p}<0.05)$.

\section{Discussion}

This study intended to compare the effects of functional strength training versus conventional physical therapy on the SVMC, gross motor function and functional balance in children with spastic diplegia. The results indicated that a six- month program of functional strength training (group II) produced better improvement in SVMC, gross motor function and functional balance in children with spastic diplegia compared with a six- month program of conventional physical therapy (group I) alone. Improvements were recorded in the two groups in all measured variables. However, higher improvements were achieved in the group II compared with the group I.

All participants followed the functional strength training and non-reported any musculoskeletal discomfort (joint pain, soreness, etc). The authors suggest that the functional progressive resistance training was convenient, accessible, practical and safe without drawback effects and is in agreement with Morton et al., [20].

The improvement recorded in the two groups can be attributed to the duration and frequency of the treatment. The statistical analysis revealed significant improvements in all measured variables after treatment in both groups as the main effect of time was statistically significant. The two groups received a treatment program which was conducted for $90 \mathrm{~min}-$ utes three-times a week for 6 successive months, which is considered as an intensive program. Trahan and Malouin [21] and Tsorlakis et al., [22] stated that increasing the frequency and duration of therapy sessions may produce significant and long-lasting changes in strength, tone, posture and gross motor performance; however, the success of any therapy depends on the commitment of the parents, caregivers, child and therapists.

The significant improvement in all measured variables after treatment recorded in favor of the group II could be attributed to the type of the exercises which consisted of a group of loaded multi-joint functional resisted exercises (sit-to-stand, half knee rise, step up) that were designed to incorporate the fundamental tasks required for the daily living performance during walking, stair climbing and general mobility. All of these activities include smooth, rhythmic motions in the sagittal, frontal and transverse planes of movement which enhanced the SVMC. This agreed with previous studies stated that, sit-to-stand, lateral step-ups, front step-ups, walking up and down stairs, high-kneeling and lateral walking are effective method to enhance motor performance, gait parameters and balance [13,23-25].

The mechanism whereby the functional strength training improved the SVMC, motor function and functional balance is likely caused by neurophysiological adaptations and microscopic alternation of muscle properties of the targeted muscles as well as neuromuscular integration and improvement of muscle strength. This explanation is supported by the findings of Fowler et al., $[5,18]$ who reported that, strength-training in individuals with CP results in neurophysiological changes such as increased co-activation of antagonist muscles, promote synergist muscles performance, enhancement of spinal cord connections and cross education bilateral effect. Those neural changes are believed to continue with training, helping 
to improve movement. Similarly, Shepherd et al., [26] mentioned that training protocols based on task-specific strength training stimulates reorganization of cortical areas of the brain being used with a task. In other words, it encompasses more than simply increasing the force-production capacity of specific muscles.

Alegre et al., [27] and Reeves et al., [28] have also illustrated that strength training conducted between 12 to 14 weeks in young and older adults have revealed increases in cross section area and thickness (hypertrophy) of the muscles. Muscle hypertrophy is the increase in the cross section of muscle due to an increase in the number (hyperplasia), or the size (hypertrophy) of muscle fibers. Additionally, Dos Santos et al., [13] and Taylor et al., [29] reported that progressive strength training is believed to affect the muscle metabolic capability and consequently, induced increase in muscle strength through morphological and metabolic acclimation. They suggested that this type of training enables the improvement of neural control commands as well as increases in strength thus, improvements in functional performance.

LeMura et al., [30] stated that limited active performance is a frequent problem in $\mathrm{CP}$ resulting in structural changes of the muscle in which type I muscle fibers (oxidative) change their structure to type II (Fast twitch). They added that, proper strength training can stimulate muscle fiber type transformation and alternations to muscle structures. In other words, fast twitch fibers might become more oxidative with proper training.

The study conducted by Kusumoto et al., [8] may support our results. They investigated the relationship between SMC of the lower extremities and knee extensors muscle strength in children with spastic diplegia. They reported significant relation between the SVMC and the maximum strength of the knee extensors.

Earlier in a randomized controlled trial conducted by Kannabiran et al., [3] who studied efficacy of functional strength training in improving gross motor function among the children with spastic diplegic CP. The results of this study showed improvement in gross motor function after 10 weeks of functional strength training program.

Our results confirm those already reported by Park et al., [31] who examined the effect of concentric and eccentric control training of the paretic leg on balance and gross motor function in children with spastic hemiplegia. They found significant improvement in gross motor function and balance ability.

Our results are consistent with Dodd et al., [23] who evaluated the effects of a home-based, six-week strength-training program on lower limb strength and physical activity of young people with spastic diplegic CP. They reported that, strength training may cause lasting changes in the strength of key lower-limb muscles that may impact on the daily function of young people with CP.

Likewise, Kannabiran et al., [3] examined the effectiveness of functional resistance training in improving motor function in preschool children with spastic diplegia. The results showed significant improvement in gross motor function particularly in standing, walking, running and jumping after 10 weeks of intervention based on functional strength training.

Additionally, Blundell et al., [26] investigated the effect of short duration functional strength training in children with CP. The concluded that, task-specific strength training conducted for short duration, caused improvement in both muscle strength and functional performance in children with CP.

The results of the current study come in agreement with Liao et al., [25] who investigated the effectiveness of loaded sit-to-stand resistance exercise for children with mild spastic diplegia. They found significant improvements of basic motor abilities, functional muscle strength, and walking efficiency.

The current study had several limitations. First, participants were delimited to children with spastic diplegia and not all subtypes of CP make it difficult to generalize the findings to all children with CP. Second, there is limited literature on the effectiveness of functional strength training on SVMC and it was difficult to affirm the study results. Hence, future researches using a larger sample and different types of $\mathrm{CP}$ are needed. Moreover, multidimensional analysis using appropriate assessment tools are recommended to validate the relation between SMC and muscle strength. Finally, studies are recommended to determine the long-term effects of muscle strengthening in children with $\mathrm{CP}$.

\section{Conclusion}

The results of the current study suggested that both the functional strength training and the conventional physical therapy have the potential to produce a significant improvement of SMC, gross motor function and functional balance in children with spastic diplegia. Moreover, the conventional physical therapy has been realized as being effective but to less extent than the functional strength training.

\section{Competing interests}

The authors declare that they have no competing interests.

Authors' contributions

\begin{tabular}{|l|c|c|}
\hline Authors' contributions & AMA & WAA \\
\hline Research concept and design & $\checkmark$ & $\checkmark$ \\
\hline Collection and/or assembly of data & $\checkmark$ & -- \\
\hline Data analysis and interpretation & $\checkmark$ & -- \\
\hline Writing the article & $\checkmark$ & $\checkmark$ \\
\hline Critical revision of the article & $\checkmark$ & $\checkmark$ \\
\hline Final approval of article & $\checkmark$ & $\checkmark$ \\
\hline
\end{tabular}

\section{Acknowledgements}

The authors would like to acknowledge the children and their legal guardians as well as all physiotherapists participated in this work for their valuable contribution in this study.

\section{Publication history}

Editor: Mohammad H. Hadadzadeh, Wheeling Jesuit University, USA. Received: 02-Mar-2019 Final Revised: 10-Apr-2019

Accepted: 18-Apr-2019 Published: 10-May-2019 


\section{References}

1. Scholtes VA, Becher JG, Comuth A, Dekkers H, Van Dijk L and Dallmeijer AJ. Effectiveness of functional progressive resistance exercise strength training on muscle strength and mobility in children with cerebral palsy: a randomized controlled trial. Dev Med Child Neurol. 2010; 52:e107-13. | Article | PubMed

2. Fernandes MV, Maifrino LBM, Monte KNS, Araújo RC, Mochizuki L and Ervilha UF. Effectiveness of resistance training exercises in spastic diplegia cerebral palsy: a review. J. Morphol Sci. 2012; 29:125-128.

3. Kannabiran B, Nandhini K and Nagarani R. A Study to Analyze the Effectiveness of Functional Strength Training in Improving Gross Motor Function among the Children with Spastic Diplegic Cerebral Palsy. Int J Neurorehabilitation. 2016; 3:239. I Article

4. Tecklin MS and Jan S. Paediatric physical therapy. Lippicott Williams and Wilkins, USA. 2015.

5. Fowler EG, Staudt LA and Greenberg MB. Lower-extremity selective voluntary motor control in patients with spastic cerebral palsy: increased distal motor impairment. Dev Med Child Neurol. 2010; 52:2649. | Article | PubMed

6. Givon U. [Muscle weakness in cerebral palsy]. Acta Orthop Traumatol Turc. 2009; 43:87-93. | Article | PubMed

7. Lim H. Correlation between the selective control assessment of lower extremity and pediatric balance scale scores in children with spastic cerebral palsy. J Phys Ther Sci. 2015; 27:3645-9. | Article | PubMed Abstract | PubMed FullText

8. Kusumoto Y, Takaki K, Matsuda T and Nitta O. Relation of selective voluntary motor control of the lower extremity and extensor strength of the knee joint in children with spastic diplegia. J Phys Ther Sci. 2016; 28:1868-71. | Article | PubMed Abstract | PubMed FullText

9. Levitt S. Treatment of Cerebral Palsy and Motor Delay: clinical picture for therapy and management, $5^{\text {th }}$ edition. John Wiley \& Sons. 2010; 6-9.

10. Galli M, Cimolin V, Pau M, Leban B, Brunner R and Albertini G. Foot pressure distribution in children with cerebral palsy while standing. Res Dev Disabil. 2015; 41-42:52-7. | Article | PubMed

11. Verschuren O, Ada L, Maltais DB, Gorter JW, Scianni A and Ketelaar M. Muscle strengthening in children and adolescents with spastic cerebral palsy: considerations for future resistance training protocols. Phys Ther. 2011; 91:1130-9. | Article | PubMed

12. Scholtes VA, Dallmeijer AJ, Rameckers EA, Verschuren O, Tempelaars E, Hensen $M$ and Becher JG. Lower limb strength training in children with cerebral palsy--a randomized controlled trial protocol for functional strength training based on progressive resistance exercise principles. BMC Pediatr. 2008; 8:41. | Article | PubMed Abstract | PubMed FullText

13. Dos Santos AN, da Costa CS, Golineleo MT and Rocha NA. Functional strength training in child with cerebral palsy GMFCS IV: case report. Dev Neurorehabil. 2013; 16:308-14. | Article | PubMed

14. Ryan JM, Theis N, Kilbride C, Baltzopoulos V, Waugh C, Shortland A, Lavelle G, Noorkoiv M, Levin W and Korff T. Strength Training for Adolescents with cerebral palsy (STAR): study protocol of a randomised controlled trial to determine the feasibility, acceptability and efficacy of resistance training for adolescents with cerebral palsy. BMJ Open. 2016; 6:e012839. | Article | PubMed Abstract | PubMed FullText

15. Sayed A and Sweif RE. Can Functional Eccentric Muscle Control Remedies Balance Ability in Children with Spastic Diplegic Cerebral Palsy. Physiother Rehabil. 2017; 2:145. | Article

16. Ansari NN, Naghdi S, Arab TK and Jalaie $S$. The interrater and intrarater reliability of the Modified Ashworth Scale in the assessment of muscle spasticity: limb and muscle group effect. NeuroRehabilitation. 2008; 23:231-7. | Article | PubMed

17. Palisano RJ, Rosenbaum P, Bartlett D and Livingston MH. Content validity of the expanded and revised Gross Motor Function Classification System. Dev Med Child Neurol. 2008; 50:744-50. | Article I PubMed

18. Fowler EG, Staudt LA, Greenberg MB and Oppenheim WL. Selective Control Assessment of the Lower Extremity (SCALE): development, validation, and interrater reliability of a clinical tool for patients with cerebral palsy. Dev Med Child Neurol. 2009; 51:607-14. | Article | PubMed

19. Badawy WM and Ibrahim MB. Balance training versus reciprocal electrical stimulation on knee joint alignment in spastic diplegic cerebral palsy children. Bulletin of Faculty of Physical Therapy. 2015; 20:146-153. | Article

20. Morton JF, Brownlee M and McFadyen AK. The effects of progressive resistance training for children with cerebral palsy. Clin Rehabil. 2005; 19:283-9. | Article | PubMed

21. Trahan $J$ and Malouin F. Intermittent intensive physiotherapy in children with cerebral palsy: a pilot study. Dev Med Child Neurol. 2002; 44:233-9. | Article | PubMed

22. Tsorlakis N, Evaggelinou C, Grouios $G$ and Tsorbatzoudis C. Effect of intensive neurodevelopmental treatment in gross motor function of children with cerebral palsy. Dev Med Child Neurol. 2004; 46:740-5. | Article I PubMed

23. Dodd KJ, Taylor NF and Graham HK. A randomized clinical trial of strength training in young people with cerebral palsy. Dev Med Child Neurol. 2003; 45:652-7. | Article | PubMed

24. Lee JH, Sung IY and Yoo JY. Therapeutic effects of strengthening exercise on gait function of cerebral palsy. Disabil Rehabil. 2008; 30:1439-44. | PubMed

25. Liao HF, Gan SM, Lin KH and Lin JJ. Effects of weight resistance on the temporal parameters and electromyography of sit-to-stand movements in children with and without cerebral palsy. Am J Phys Med Rehabil. 2010; 89:99-106. | Article | PubMed

26. Blundell SW, Shepherd RB, Dean CM, Adams RD and Cahill BM. Functional strength training in cerebral palsy: a pilot study of a group circuit training class for children aged 4-8 years. Clin Rehabil. 2003; 17:48-57. | Article | PubMed

27. Alegre LM, Jimenez F, Gonzalo-Orden JM, Martin-Acero R and Aguado X. Effects of dynamic resistance training on fascicle length and isometric strength. J Sports Sci. 2006; 24:501-8. | Article | PubMed

28. Reeves ND, Maganaris CN, Longo $S$ and Narici MV. Differential adaptations to eccentric versus conventional resistance training in older humans. Exp Physiol. 2009; 94:825-33. | Article | PubMed

29. Taylor NF, Dodd KJ and Damiano DL. Progressive resistance exercise in physical therapy: a summary of systematic reviews. Phys Ther. 2005; 85:1208-23. | PubMed

30. LeMura LM and von Duvillard SP. Clinical Exercise Physiology. Application and Physiological Principles. Philadelphia: Lippincott Williams \& Wilkins. 2004: 235-8

31. Park SI, Kim MS and Choi JD. Effects of concentric and eccentric control exercise on gross motor function and balance ability of paretic leg in children with spastic hemiplegia. J Phys Ther Sci. 2016; 28:2128-31. I Article | PubMed Abstract | PubMed FullText

\section{Citation:}

Abd-Elmonem AM and Abd El-nabie WA. Therapeutic Outcomes Of Functional Strength Training Versus Conventional Physical Therapy In Children With Cerebral Palsy: A Comparative Study. Phys Ther Rehabil. 2019; 6:7. http://dx.doi.org/10.7243/2055-2386-6-7 